\section{Características clínicas del trastorno depresivo mayor en tratamiento en el nivel secundario de atención}

\author{
LILIAN SALVO ${ }^{1,2,3, a}$, SANDRA SALDIVIA ${ }^{4, b, c}$, CARLOS PARRA $^{3,5,6}$, \\ ROMÁN RODRÍGUEZ ${ }^{3,6}$, MANUEL CIFUENTES $^{7, \mathrm{~d}}$, \\ PAOLA ACEVEDO ${ }^{3, \mathrm{~b}}$, MARCELA DÍAZ $^{3, \mathrm{~b}}$, MITZA ORMAZABAL $^{5, \mathrm{~b}}$, \\ IVONNE GUERRA ${ }^{5, \mathrm{~b}}$, NICOL NAVARRETE6, \\ VERÓNICA BRAVO ${ }^{6, b}$, ANDREA CASTRO $^{\mathrm{b}}$
}

\section{Clinical features of major depressive disorders treated in secondary health care facilities in Chile}

Background: Depression is considered the second leading cause of disability worldwide. Aim: To describe the clinical characteristics and the evolution of major depressive disorder (MDD) in secondary care. To evaluate the association between socio-demographic and clinic variables with the first or recurrent major depressive events (MDE). Material and Methods: Clinical features, treatment, remission and duration of MDE were evaluated during a follow up lasting 12 months in 112 participants aged $44 \pm 15$ years ( $79 \%$ women). Patients were assessed as outpatients every three months at three psychiatric care centers of Chile. Clinical interviews were carried out using DSM-IV diagnostic criteria checklists and the Hamilton Depression Scale was applied. Results: Most patients were referred from primary care. The mean time lapse for referral to the secondary level was 10.8 months. Most patients had episodes that were recurrent, severe, with a high rate of psychosis, with suicide attempts and melancholic features and with psychiatric and medical comorbidities. Remission rate was $27.5 \%$. In only $16 \%$ of patients, the episode lasted six months or less. The group with recurrent episodes had different age, sex and clinical features. Conclusions: MDD treated at the secondary care level is severe and its symptoms are intense. The time lapse prior to referral was prolonged. Primary care management and referral of these patients should be studied more closely.

(Rev Med Chile 2017; 145: 335-343)

Key words: Depression; Outpatients; Public Health; Secondary Care.

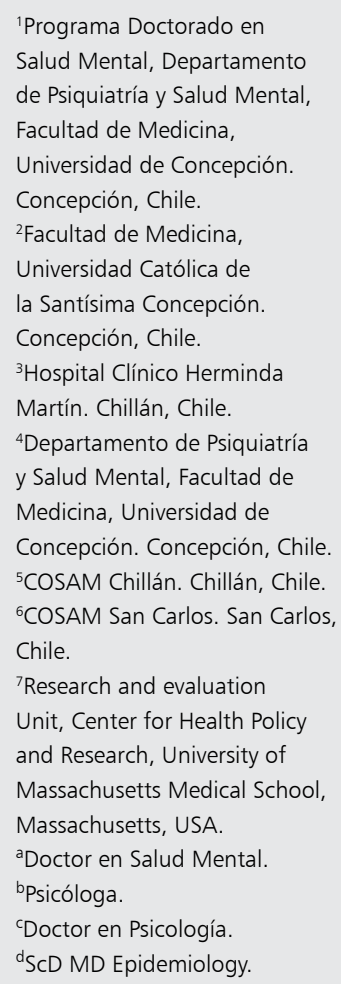

Fuente de apoyo financiero: El presente artículo no contó con apoyo financiero.

Recibido el 24 de mayo 2016, aceptado el 6 de marzo de 2017 .

Correspondencia a:

Lilian Salvo G. Bulnes 473,

Chillán.

Isalvog@gmail.com
L a depresión constituye un problema para la salud pública en el mundo y en Chile. Al valorar la carga de enfermedad, la Organización Mundial de la Salud, el año 2010, estimó que el trastorno depresivo mayor (TDM) ocupó el lugar número 11, como causa de más discapacidad y mortalidad; a la vez, la consideró la segunda causa de discapacidad en el mundo ${ }^{1,2}$. Asimismo, es responsable de más de $40 \%$ de las alteraciones de las capacidades causadas por los trastornos mentales ${ }^{3,4}$. Por lo tanto, es evidente que se deben desplegar diversas estrategias de afrontamiento en todos los niveles de atención.

Se ha demostrado que la carga de enfermedad causada por el TDM puede ser reducida si se aumenta la cobertura del tratamiento ${ }^{5}$. Pero, se 
requiere que éste sea efectivo, para lo cual es necesario, entre muchos otros factores, contar con un diagnóstico clínico adecuado.

En nuestro país se han llevado a cabo varios estudios en atención primaria, pero escasas investigaciones se han efectuado en el nivel secundario ${ }^{6,7}$.

El objetivo de este estudio es describir las características clínicas y la evolución del TDM, y evaluar la relación de variables sociodemográficas y clínicas con primer EDM versus recurrentes, en personas tratadas en el nivel secundario de atención del Servicio de Salud de Ñuble (SSÑ).

\section{Material y Método}

Se trata de un estudio no experimental, longitudinal, con seguimiento durante doce meses. La población de estudio la constituyen todas las personas con diagnóstico de TDM realizado por psiquiatra, que iniciaron tratamiento en los tres centros de atención secundaria del SSÑ entre el 01/08/2013 y el $31 / 07 / 2014$, que tenían 15 y más años de edad. Los criterios de exclusión fueron: trastorno bipolar, dependencia a sustancias descompensada y enfermedad médica o condición discapacitante que imposibilitara las evaluaciones. A quienes aceptaron participar se les realizó evaluación basal y seguimiento por 12 meses, con evaluaciones cada 3 meses. Cumplieron los criterios de inclusión 121 pacientes; 6 no aceptaron participar y 3 fueron excluidos durante el seguimiento por cambio de diagnóstico. La muestra quedó constituida por 112 pacientes y 102 completaron el seguimiento. Todos fueron entrevistados individualmente por psiquiatra en la evaluación basal y por psicóloga en las evaluaciones siguientes.

El episodio depresivo mayor (EDM) y sus diversas características fueron definidas siguiendo los criterios del DSM-IV y evaluadas en entrevista clínica psiquiátrica, se usó checklist de criterios diagnósticos DSM-IV ${ }^{8}$. La remisión, (parcial o total según DSM-IV $)^{8}$ fue evaluada clínicamente por psiquiatra. Además, fue medida con la Escala de Depresión de Hamilton (Ham-D) ${ }^{9,10}$. En concordancia con los acuerdos para investigación, se consideró sin remisión al puntaje total en Ham-D $\geq 18$, remisión parcial entre 8 y 17 puntos, y remisión total al puntaje $\leq 7^{11,12}$. La duración del EDM con tratamiento en el nivel secundario fue definida como el tiempo entre el inicio del trata- miento en ese nivel y la remisión total mantenida por dos meses. El tiempo de derivación se obtuvo de documentos de referencia. El tratamiento fue extraído de la historia clínica.

La entrevista psiquiátrica con checklist de criterios diagnósticos DSM-IV es una entrevista clínica basada en los criterios diagnósticos de dicho manual ${ }^{8}$. La escala Ham-D (versión 17 ítems) evalúa el perfil sintomático y gravedad de la depresión ${ }^{9,10}$. En ensayos clínicos el punto de corte más utilizado es $\leq 7$ : sin depresión; también empleado en Chi$1 \mathrm{e}^{6,13}$. Su validez ha sido demostrada en nuestro medio $^{14-16}$. En este estudio, obtuvo un Coeficiente alfa de Cronbach de 0,77.

El estudio fue aprobado por los Comités de Ética de la Universidad de Concepción y SSÑ. Se coordinó la obtención de registros en cada centro de salud. Identificado el ingreso, se contactó personalmente al paciente y se invitó a participar. Quienes aceptaron firmaron el consentimiento informado para el estudio y para obtener información de su historia clínica.

Los datos se analizaron con el programa estadístico SPSS V21. En este artículo se presentan resultados de análisis descriptivos y bivariados (prueba t de Student, chi cuadrado y estadístico exacto de Fisher) para evaluar la relación de variables sociodemográficas y clínicas con primer episodio versus episodios recurrentes. Se estableció nivel de significación de 0,05.

\section{Resultados}

\section{Características sociodemográficas}

El promedio de edad fue 43,9 años (DE: 15,3), el 13,4\% tenía entre 15 y 19 años. La mayoría eran mujeres casadas, vivían con su pareja, tenían estudios medios y eran dueñas de casa. Aproximadamente un cuarto contaba con trabajo remunerado y un quinto provenía del medio rural. En cuanto a las características socio-demográficas según primer episodio versus recurrente, sólo se encontró asociación significativa con edad y sexo; la subpoblación con EDM recurrentes tenía mayor edad que la con un primer EDM y el porcentaje de mujeres con EDM recurrente fue superior al de los hombres (Tabla 1).

\section{Características clínicas}

En la mayoría el EDM era recurrente, grave (por clínica y Ham-D) y sin remisión. Aproxima- 
Tabla 1. Características sociodemográficas de los pacientes con TDM en tratamiento en el nivel secundario del Servicio de Salud de Ñuble y su relación con primer episodio versus episodio recurrente

\begin{tabular}{|c|c|c|c|c|c|c|}
\hline \multirow[t]{2}{*}{ Variable } & \multicolumn{2}{|c|}{ Primer EDM } & \multicolumn{2}{|c|}{ EDM recurrente } & \multicolumn{2}{|c|}{ Total } \\
\hline & n (45) & $\%$ & n (67) & $\%$ & n (112) & $\%$ \\
\hline Edad (años) ${ }^{a *} \quad M(D S)$ & 40,9 & $(16,8)$ & 46 & $(14,1)$ & 43,9 & $(15,3)$ \\
\hline \multicolumn{7}{|l|}{ Sexo ${ }^{b *}$} \\
\hline Masculino & 14 & 31,1 & 10 & 14,9 & 24 & 21,4 \\
\hline Femenino & 31 & 68,9 & 57 & 85,1 & 88 & 78,6 \\
\hline \multicolumn{7}{|l|}{ Estado civil ${ }^{\mathrm{c}}$} \\
\hline Soltera(o) & 16 & 35,6 & 20 & 29,9 & 36 & 32,1 \\
\hline Casada(o) & 15 & 33,3 & 24 & 35,8 & 39 & 34,8 \\
\hline Conviviente & 2 & 4,4 & 5 & 7,5 & 7 & 6,3 \\
\hline Separada/divorciada(o) & 10 & 22,2 & 12 & 17,9 & 22 & 19,6 \\
\hline Viuda(o) & 2 & 4,4 & 6 & 9,0 & 8 & 7,2 \\
\hline \multicolumn{7}{|l|}{ Vive $\operatorname{con}^{c}$} \\
\hline Con sus padres & 12 & 26,7 & 8 & 11,9 & 20 & 17,8 \\
\hline Con su pareja con/sin hijos & 15 & 33,3 & 31 & 46,3 & 46 & 41,1 \\
\hline Con hijos y otros familiares & 1 & 2,2 & 2 & 3,0 & 3 & 2,7 \\
\hline Sola con sus hijos & 6 & 13,3 & 15 & 22,4 & 21 & 18,8 \\
\hline Sola(o) & 6 & 13,3 & 4 & 6,0 & 10 & 8,9 \\
\hline Otra situación & 5 & 11,1 & 7 & 10,4 & 12 & 10,7 \\
\hline \multicolumn{7}{|l|}{ Escolaridad } \\
\hline Sin escolaridad & 2 & 4,4 & - & - & 2 & 1,8 \\
\hline Básica & 14 & 31,1 & 25 & 37,3 & 39 & 34,8 \\
\hline Media & 18 & 40,0 & 24 & 35,8 & 42 & 37,5 \\
\hline Técnica & 4 & 8,9 & 7 & 10,4 & 11 & 9,8 \\
\hline Universitaria & 7 & 15,6 & 11 & 16,4 & 18 & 16,1 \\
\hline \multicolumn{7}{|l|}{ Estado de empleo ${ }^{b}$} \\
\hline Con trabajo remunerado & 10 & 22,2 & 23 & 34,3 & 33 & 29,5 \\
\hline Sin trabajo & 10 & 22,2 & 9 & 13,4 & 19 & 16,9 \\
\hline Jubilado & 2 & 4,4 & 7 & 10,4 & 9 & 8,0 \\
\hline Dueña de casa & 13 & 28,9 & 21 & 31,3 & 34 & 30,4 \\
\hline Estudiante & 10 & 22,2 & 7 & 10,7 & 17 & 15,2 \\
\hline \multicolumn{7}{|l|}{ Procedencia $^{b}$} \\
\hline Urbano & 35 & 77,8 & 53 & 79,1 & 88 & 78,6 \\
\hline Rural & 10 & 22,2 & 14 & 20,9 & 24 & 21,4 \\
\hline
\end{tabular}

${ }^{a}=$ t Student; ${ }^{b}=\chi^{2} ;{ }^{c}=$ Estadístico Exacto de Fisher. ${ }^{*} p<0,05$

damente un tercio presentó síntomas psicóticos y cerca de la mitad, intentó suicidarse. Un 57,1\% presentó psicosis y/o intento suicida. Un alto porcentaje tenía comorbilidad psiquiátrica, siendo la distimia, el trastorno relacionado con sustancias y el trastorno de ansiedad, los más frecuentes en eje I. Se diagnosticó trastorno de personalidad en dos tercios de los casos. La mayoría presentaba enfermedad médica; las más frecuentes fueron: hipertensión arterial, dislipidemia, hipotiroidismo y dolor crónico (Tabla 2).
La relación entre las características clínicas estudiadas y las subpoblaciones de primer episodio versus recurrente, resultó significativa sólo para gravedad basal, intento suicida y cronicidad. Los pacientes con episodios recurrentes presentaron mayor puntaje en Ham-D en comparación con los con episodio único; $y$ los con un primer episodio tenían mayor porcentaje de intentos suicidas y episodios crónicos que los con EDM recurrentes. Los síntomas psicóticos, características melancólicas, comorbilidad psiquiátrica y comorbilidad 
médica fueron más frecuentes en los pacientes con episodios recurrentes que en los con un primer episodio, pero no alcanzaron significación estadística (Tabla 2).

El 73,2\% (n: 82) fue referido desde atención primaria. El tiempo previo a tratamiento en el nivel secundario fue en promedio 10,8 meses (DE: 14,7). Los pacientes con intento de suicidio se derivaron en promedio a los 11,2 meses (DE: 2,1) y con síntomas psicóticos a los 11,4 meses (DE: 2,3).

Tabla 2. Características clínicas de los pacientes con TDM al ingreso a tratamiento en el nivel secundario del Servicio de Salud de Ñuble y su relación con primer episodio versus episodio recurrente

\begin{tabular}{|c|c|c|c|c|c|c|}
\hline \multirow[t]{2}{*}{ Variable } & \multicolumn{2}{|c|}{ Primer EDM } & \multicolumn{2}{|c|}{ EDM recurrente } & \multicolumn{2}{|c|}{ Total } \\
\hline & n (45) & $\%$ & n (67) & $\%$ & n (112) & $\%$ \\
\hline Puntaje Ham-D ${ }^{a *} \quad M(D E)$ & 28,8 & $(7,2)$ & 31,6 & $(6,4)$ & 30,5 & $(6,8)$ \\
\hline \multicolumn{7}{|l|}{ Gravedad $^{\mathrm{b}}$} \\
\hline EDM grave & 38 & 84,4 & 56 & 83,6 & 94 & 83,9 \\
\hline EDM moderado & 7 & 15,6 & 11 & 16,4 & 18 & 16,1 \\
\hline \multicolumn{7}{|l|}{ Remisión por clínica } \\
\hline EDM sin remisión & 43 & 95,6 & 67 & 100,0 & 110 & 98,2 \\
\hline EDM en remisión parcial & 2 & 4,4 & - & - & 2 & 1,8 \\
\hline \multicolumn{7}{|l|}{ Características del EDM ${ }^{\mathrm{b}}$} \\
\hline Síntomas psicóticos ${ }^{b}$ & 13 & 28,9 & 23 & 34,3 & 36 & 32,1 \\
\hline Intento de suicidio ${ }^{\mathrm{b} *}$ & 25 & 55,6 & 24 & 35,8 & 49 & 43,8 \\
\hline Características melancólicas ${ }^{b}$ & 16 & 35,6 & 28 & 41,8 & 44 & 39,3 \\
\hline Características catatónicas & - & - & 2 & 3,0 & 2 & 1,8 \\
\hline Características atípicas ${ }^{c}$ & 11 & 24,4 & 16 & 23,9 & 27 & 24,1 \\
\hline Crónico ${ }^{\mathrm{b} *}$ & 17 & 37,8 & 13 & 19,4 & 30 & 26,8 \\
\hline \multicolumn{7}{|l|}{ Psicosis- Intento suicida ${ }^{b}$} \\
\hline Sin psicosis ni intentos suicidas & 17 & 37,8 & 31 & 46,3 & 48 & 42,9 \\
\hline Con síntomas psicóticos & 3 & 6,7 & 12 & 17,9 & 15 & 13,3 \\
\hline Con intentos suicidas & 15 & 33,3 & 13 & 19,4 & 28 & 25,0 \\
\hline Con psicosis e intentos suicidas & 10 & 22,2 & 11 & 16,4 & 21 & 18,8 \\
\hline Comorbilidad psiquiátricab $^{b}$ & 34 & 75,6 & 54 & 80,6 & 88 & 78,6 \\
\hline En eje I DSM-IVb & 24 & 53,3 & 27 & 40,3 & 51 & 45,5 \\
\hline Distimia $^{\mathrm{b}}$ & 18 & 40,0 & 17 & 25,4 & 35 & 31,3 \\
\hline T. relacionados con sustancias & 4 & 8,9 & 5 & 7,5 & 9 & 8,0 \\
\hline T. por ansiedad & 2 & 4,4 & 3 & 4,5 & 5 & 4,5 \\
\hline Otros & 6 & 13,3 & 3 & 4,5 & 9 & 8,0 \\
\hline T. de personalidad ${ }^{b}$ & 29 & 64,4 & 45 & 67,2 & 74 & 66,1 \\
\hline Grupo A & 1 & 2,2 & 1 & 1,5 & 2 & 1,8 \\
\hline Grupo B & 6 & 13,3 & 13 & 19,4 & 19 & 17,0 \\
\hline Grupo C & 6 & 13,3 & 13 & 19,4 & 19 & 17,0 \\
\hline Grupo B y C & 8 & 17,8 & 9 & 13,4 & 17 & 15,2 \\
\hline No especificado & 9 & 20,0 & 8 & 11,9 & 17 & 15,2 \\
\hline Comorbilidad médicab & 26 & 57,8 & 48 & 71,6 & 74 & 66,1 \\
\hline Hipotiroidismo & 5 & 11,1 & 15 & 22,4 & 20 & 17,9 \\
\hline Hipertensión arterial & 12 & 26,7 & 16 & 23,9 & 28 & 25,0 \\
\hline Dislipidemia & 6 & 13,3 & 17 & 25,4 & 23 & 20,5 \\
\hline Diabetes Mellitus & 5 & 11,1 & 8 & 11,9 & 13 & 11,6 \\
\hline Dolor crónico & 5 & 11,1 & 9 & 13,4 & 14 & 12,5 \\
\hline Otras & 18 & 40,0 & 26 & 38,8 & 54 & 48,2 \\
\hline
\end{tabular}

${ }^{a}=$ t Student; ${ }^{b}=\chi^{2} ;{ }^{c}=$ Estadístico Exacto de Fisher. ${ }^{*} p<0,05$. EDM: episodio depresivo mayor. T: Trastorno. 


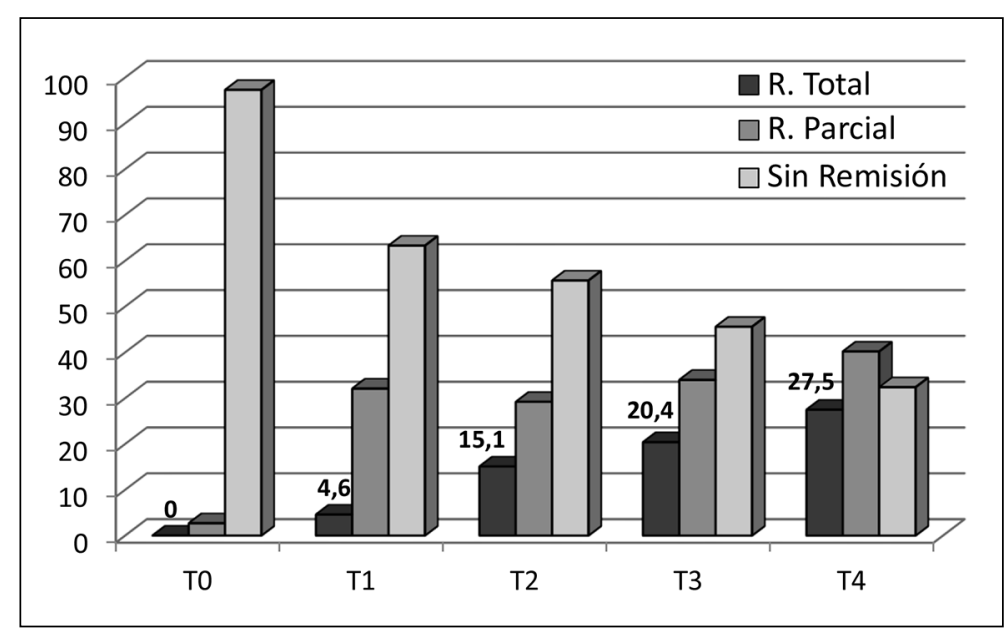

Figura 1. Remisión del EDM en pacientes con trastorno depresivo mayor en tratamiento en el nivel secundario del Servicio de Salud de Ñuble.
Durante el seguimiento 4 pacientes intentaron suicidarse, ninguno consumó el suicidio y 2 fueron excluidos del estudio por presentar episodio maniaco.

\section{Tratamiento}

Al revisar la historia clínica se encontró que al 92\% (n: 103) se le indicó antidepresivos, generalmente junto a otro psicofármaco: más neurolépticos y benzodiazepinas $(23,2 \%)$, más neurolépticos $(22,3 \%)$, y más estabilizadores y neurolépticos (15,2\%). Un 8\% (n: 9) no recibió antidepresivos, pero sí estabilizadores del ánimo. Los antidepresivos más recetados fueron: venlafaxina $(33,0 \%)$ y sertralina $(32,1 \%)$; estabilizadores del ánimo: lamotrigina $(12,5 \%)$ y ácido valproico $(8,9 \%)$; neurolépticos: quetiapina $(45,5 \%)$ y risperidona $(24,1 \%)$; benzodiazepinas: clonazepam $(54,5 \%)$ y alprazolam $(1,8 \%)$; otros psicofármacos: disulfiram y propanolol (3,6\% cada uno).

En cuanto a las intervenciones psicoterapéuticas, realizadas por psicóloga(o), el 98,2\% (n: 110) recibió una o más intervenciones psicoterapéuticas; en promedio 5,8 (DE: 3,3) sesiones, en promedio cada 41,7 días (DE: 26,1). Sólo el 5,9\% (n: 6) recibió 6 o más sesiones con frecuencia menor o igual a 21 días.

El 82,1\% (n: 92) recibió una o más intervenciones socio-familiares por asistente social, con promedio de 3,3 (DE: 2,8) intervenciones. Un 14,3\% (n: 16) fue intervenido por dupla psico-social, con promedio de 1,5 (DE: 0,2) sesiones.

\section{Remisión y duración del EDM}

En la evaluación basal (T0) el puntaje Ham-D promedio fue de 30,5 puntos (DE 6,8) y a los 12 meses (T4) fue de 13,3 (DE 7,4). Se observó una mayor caída del puntaje a los 3 meses (11 puntos). Posteriormente el descenso fue menos abrupto (2 puntos cada tres meses). Respecto al ingreso, a los doce meses el descenso fue de $56,4 \%$. Al término del seguimiento se encontró $27,5 \%$ de remisión total, $40,2 \%$ de remisión parcial y $32,3 \%$ aún estaba en EDM sin remisión (Figura 1).

En el $72,5 \%$ de los participantes el episodio duró más de 12 meses. En el 15,7\% el episodio duró 6 o menos meses. En los pacientes con remisión total, la duración promedio del EDM fue de 6,6 meses (DE: 3,6).

\section{Discusión}

El actual programa nacional de depresión no ha sido evaluado en sus resultados. Este estudio provee información inicial sobre la evolución del TDM en el nivel secundario.

El porcentaje de episodios recurrentes encontrado es concordante con lo reportado por Retamal en Chile el $2004(56 \%)^{6}$ y lo publicado en estudios internacionales $(37 \% \text { a } 85 \%)^{17}$.

De acuerdo con el puntaje basal en Ham-D (30,5 puntos), nuestros pacientes presentaron EDM más graves que los participantes de un estudio nacional $(23,4 \text { puntos })^{6}$, del STAR ${ }^{\star} \mathrm{D}(21,8$ 
puntos $)^{18}$ y del estudio Vantaa (18,6 puntos $)^{19}$. Se ha demostrado que los EDM más graves tienen peor remisión, recurrencia, cronicidad y respuesta a tratamiento ${ }^{20-23}$.

La prevalencia de depresión psicótica es variable: 15 a $20 \%$ en población general ${ }^{24}$ y 24 a $53 \%$ en hospitalizados ${ }^{25}$; por tanto, nuestras cifras $(32,1 \%)$ son aceptables por tratarse de episodios graves y atención especializada. Pero estos síntomas psicóticos también podrían constituir señales de una posible depresión bipolar. Si consideramos dos pacientes con viraje a manía durante el seguimiento, el porcentaje de psicosis, la edad de inicio precoz $(13,4 \%)$ y el porcentaje que recibió estabilizadores con o sin antidepresivos: debemos reevaluar la muestra en busca de cuadros del espectro bipolar, incluyendo además otras características que orienten en ese sentido, como historia familiar de bipolaridad, anhedonia severa, cambios de humor estacionales e inicio en post parto.

El porcentaje de intentos de suicidio fue superior a lo reportado en centros especializados ambulatorios ${ }^{6,18,19}$. En nuestro país se recomienda derivar pacientes con TDM desde atención primaria si presentan psicosis y/o intentos suicidas ${ }^{26}$. Llama la atención la extensión del tiempo de derivación en estos casos (11,4 y 11,2 meses respectivamente). A la luz de estos resultados se requiere revisar la calificación de la gravedad y oportunidad de la derivación hacia el nivel secundario, más aún frente a dichos síntomas por su implicancia en el tratamiento, evolución y riesgo de suicidio. La dificultad en la clasificación de severidad del EDM en atención primaria ya se había informado el $2011^{27}$. Por lo anterior, es una necesidad urgente mejorar la capacitación de nuestros médicos generales.

Las características melancólicas están presentes en el 25 al 30\% de los $\mathrm{EDM}^{28}$. El porcentaje encontrado es ligeramente superior y concordante con la severidad. Estas características son importantes de evaluar por su relación con riesgo suicida, recurrencia y menor respuesta terapéutica ${ }^{20,28-32}$. El porcentaje de síntomas catatónicos y características atípicas se enmarcan dentro de lo descrito ${ }^{30,33}$. Dado que la muestra está conformada por pacientes graves y/o con falta de respuesta terapéutica, se esperaba este porcentaje de EDM crónicos $(26,8 \%)$ superior al de la población general $(15 \%)^{34}$.

Como en otros estudios, nuestros pacientes presentaron alto porcentaje de comorbilidad psiquiátrica $^{18,19}$. Sin embargo, encontramos bajas cifras de trastornos ansiosos $(4,5 \%$ versus 24 a $41 \%)$; probablemente su diagnóstico esté subestimado o se consideró como depresión con características ansiosas, entre otras posibles explicaciones que requieren ser profunizadas. La comorbilidad psiquiátrica aumenta el riesgo de severidad y persistencia, a la vez que representa un mayor desafío terapéutico ${ }^{20-22,32,35,36}$.

Respecto a las condiciones médicas, la mayor frecuencia de trastornos cardiovasculares, metabólicos y dolorosos refleja lo que ocurre en la población general ${ }^{37}$. Las cifras de hipotiroidismo podrían explicarse por su asociación con $\mathrm{TDM}^{38,39}$. Es sabido que en personas con condiciones médicas crónicas la prevalencia de TDM es más alta ${ }^{37}$ y que la co-ocurrencia con TDM se relaciona con peores resultados en ambos sentidos ${ }^{40}$.

La gran mayoría de los pacientes presentó EDM graves y complejos, por tanto el tiempo previo a tratamiento en el nivel secundario $(10,8$ meses) es muy prolongado y requiere ser evaluado; aún más si se considera que en nuestro país el programa de garantías explícitas en salud para la depresión ${ }^{26,41}$ establece 30 días como tiempo máximo para otorgar una hora de evaluación por psiquiatra.

Se esperaba un predominio significativo de desventajas sociodemográficas en la subpoblación con EDM recurrentes: el no observarse podría ser explicado por el tamaño de la muestra. Esta misma explicación podría dar cuenta de la falta de significación estadística para la mayor frecuencia de características clínicas que implican gravedad y complejidad en los pacientes con episodios recurrentes que en los con primer episodio.

En general no se observó un patrón determinado respecto a la prescripción de fármacos. Sería interesante evaluar si el porcentaje que no recibió antidepresivos y sí estabilizadores del ánimo corresponde a trastorno bipolar o TDM con características mixtas ${ }^{42,43}$. Las combinaciones farmacológicas observadas pueden explicarse por la gravedad y complejidad del TDM. Un alto porcentaje recibió ansiolíticos; cabe preguntarse si los síntomas ansiosos corresponden a un trastorno ansioso no diagnosticado o a TDM con ansiedad ${ }^{8}$. Es un desafío a futuro evaluar la implementación y cumplimiento de la farmacoterapia de acuerdo a las guías clínicas.

En los distintos centros no se efectuó psicoterapia con efectividad demostrada en $\mathrm{TDM}^{32,44}$. 
Las intervenciones psicoterapéuticas fueron realizadas por psicólogas(os) con distintos años de experiencia clínica. Dada la complejidad de los pacientes en este nivel, se hace necesario favorecer la capacitación de estos profesionales.

La tasa de remisión total $(27,5 \%)$ fue similar a la etapa I del $\operatorname{STAR}^{\star} \mathrm{D}$. Nuestros pacientes se asemejan a etapas I, II y III, donde ellos alcanzaron remisión muy superior ${ }^{18}$. Ciudad ${ }^{45}$ reportó $20,5 \%$ de remisión total a las 6 semanas, pero su muestra incluía pacientes menos graves, sin psicosis ni comorbilidad psiquiátrica. Novick ${ }^{23}$ informó $78,6 \%$ de remisión total a los 6 meses; pero utilizó un inventario de autoreporte de sintomatología depresiva, no incluyó TDM con psicosis, depresión resistente, distimia, deterioro cognitivo ni discapacidad mental. El estudio Vantaa ${ }^{19}$, con 5 años de seguimiento, reportó $49 \%$ de remisión total. Comparar las tasas de remisión es difícil cuando los criterios de inclusión no son similares; sin embargo, las cifras demuestran que, independiente de los centros, usando fármacos de la misma generación, el tratamiento logra resultados con un techo de respuesta.

Al término de nuestro seguimiento 32,4\% continuaba en EDM sin remisión y en el estudio finlandés ${ }^{19}$ a los cinco años $20 \%$ permanecía cumpliendo criterios para dicho episodio. Si bien nuestro estudio es corto en seguimiento para recurrencia y cronicidad, la tendencia de la remisión más lo publicado por otros autores reafirman la cronicidad y menor respuesta terapéutica en un grupo de pacientes ${ }^{19,21,34,46}$.

En nuestra muestra, a los 12 meses de tratamiento, la mayoría no había logrado remisión total y presentaba episodios de larga duración, condiciones que ensombrecen el pronóstico y se convierten en factores de riesgo para mayor duración en un nuevo $\mathrm{EDM}^{47,48}$. Para disminuir la carga de enfermedad asociada al TDM así como su impacto a nivel funcional personal, familiar y social se debe continuar insistiendo en estrategias que acorten la duración de estos episodios, por tanto que mejoren el acceso y la consulta precoz, el diagnóstico y el tratamiento adecuado en el nivel primario, la referencia oportuna a los centros especializados y un tratamiento efectivo en este nivel ${ }^{49}$.

El tratarse de un diseño observacional es una de las limitaciones del estudio. Queda pendiente la presentación de resultados de análisis multivariados.

\section{Referencias}

1. Murray CJL, Vos T, Lozano R, Naghavi M, Flaxman AD, Michaud C, et al. Disability-adjusted life years (DALYs) for 291 diseases and injuries in 21 regions, 1990-2010: a systematic analysis for the Global Burden of Disease Study 2010. Lancet 2012; 15: 380 (9859): 2197-223.

2. Ferrari AJ, Charlson FJ, Norman RE, Patten SB, Freedman G, Murray CJL, et al. Burden of depressive disorders by country, sex, age, and year: findings from the global burden of disease study 2010. PLoS medicine 2013; 10 (11): e1001547.

3. Whiteford HA, Degenhardt L, Rehm J, Baxter AJ, Ferrari AJ, Erskine HE, et al. Global burden of disease attributable to mental and substance use disorders: findings from the Global Burden of Disease Study 2010. Lancet 2013; 9904 (382): 1575-86.

4. Kessler R. The cost of depression. Psychiatr Clin N Am 2012; 35: 1-14.

5. Chisholm D, Sanderson K, Ayuso-Mateos JL, Saxena S. Reducing the global burden of depression: a population-level analysis of intervention cost-effectiveness in 14 pidemiologically-defined sub-regions (WHO CHOICE). Br J Psychiatry 2004; 184: 393-403.

6. Retamal P, Florenzano R, Vacarezza A, Chamorro X, Armijo I, Leighton C, et al. Evaluación del tratamiento de depresión severa: un estudio en un centro especializado de referencia: Rev Latinoamericana Psiquiatría 2006; 6: 18-24.

7. Vitrol V, Cancino A, Florenzano R, Ballesteros S, Schwartz D. Eficacia y costos asociados a un tratamiento ambulatorio en mujeres con depresión severa y trauma temprano. Rev Med Chile 2010; 138: 428-36.

8. American Pychiatric Association. Manual Diagnóstico y Estadístico de los Trastornos Mentales DSM-5. 5a edición. Madrid: Editorial Médica Panamericana; 2014.

9. Hamilton M. A rating scale for depression. J Neurol Neurosurg Psychiatry 1960; 23: 56-62.

10. Williams JBW. A structured interview guide for the Hamilton Depression Rating Scale. Arch Gen Psychiatry 1988, 45: 742-7.

11. Frank E, Prien RF, Jarrett RB, Keller MB, Kupfer DJ, Lavori PW, et al. Conceptualization and rationale for consensus definitions of terms in major depressive disorder. Remission, recovery, relapse, and recurrence. Arch Gen Psychiatry 1991; 48 (9): 851-5.

12. Paykel ES, Ramana R, Cooper Z, Hayhurst H, Kerr J, Barocka A. Residual symptoms after partial remission: an important outcome in depression. Psychol Med 1995; 25: 1171-80. 
13. Tranter R, O’Donovan C, Chandarana P, Kennedy S. Prevalence and outcome of partial remission in depression. J Psychiatry Neurosci 2002; 27 (4): 241-7.

14. Ruiz H, Silva H, Miranda E. Clinical and psychometric diagnosis of depression in general medical patients. Rev Med Chile 2001; 129 (6): 627-33.

15. Ojeda C, Silva H, Cabrera J, Tchimino C, De la Carrera C, Molina A. Validez interna y concurrente de una escala breve autoadministrada de siete ítems para la depresión, evaluada en relación a los inventarios de Beck y Hamilton. Estudio preliminar. Rev Chil Neuro-Psiquiat 2003; 41 (1): 31-8.

16. Baader T, Molina J, Venezian S, Rojas C, Farías R, Fierro-Freixeneta $\mathrm{C}$, et al. Validación y utilidad de la encuesta PHQ-9 (Patient Health Questionnaire) en el diagnóstico de depresión en pacientes usuarios de atención primaria en Chile. Rev Chil Neuro-Psiquiat 2012; 50 (1): 10-22.

17. Hardeveld F, Spijker J, De Graaf R, Nolen W, Beekman A. Prevalence and predictors of recurrence of major depressive disorder in the adult population. Acta Psychiatr Scand 2010; 122: 184-91.

18. Trivedi MH, Rush AJ, Wisniewski SR, Nierenberg AA, Warden D, Ritz L, et al. Evaluation of outcomes with citalopram for depression using measurement-based care in $\mathrm{STAR}^{\star} \mathrm{D}$ : implications for clinical practice. Am J Psychiatry 2006; 163 (1): 28-40.

19. Holma KM, Holma IA, Melartin TK, Rytsala HJ, Isometsa ET. Long-term outcome of major depressive disorder in psychiatric patients is variable. J Clin Psychiatry 2008; 69: 196-205.

20. Anderson IM, Ferrier IN, Baldwin RC, Cowen PJ, Howard L, Lewis G, et al. Evidence -based guidelines for treating depressive disorders with antidepressants: A revision of the 2000 British Association for Psychopharmacology guidelines. J Psychopharmacol 2008; 22: 34396.

21. Wang J, Patten S, Currie S, Sareen J, Schmitz N. Predictors of 1-year outcomes of major depressive disorder among individuals with a lifetime diagnosis: a population-based study. Psychol Med 2012; 42: 327-34.

22. Cayún G, Cantrell R, Zarotsky V, Haynes V, Phillips G, Alatorre $\mathrm{C}$, et al. Comprehensive review of factors implicated in the heterogeneity of response in depression. Depress anxiety 2012; 29: 340-54.

23. Novick D, Hong J, Montgomery W, Dueñas H, Gado M, Haro JM. Predictors of remission in the treatment of major depressive disorder: real-world evidence from a 6-month prospective observational study. Neuropsychiatr Dis Treat 2015; 11: 197-205.

24. Ohayon MM, Schatzberg AF. Prevalence of depressive episodes with psychotic features in the general population. Am J Psychiatry 2002; 159: 1855-61.

25. Gournellis R, Lykouras L. Psychotic (delusional) major depression in the elderly: A review. Curr Psychiatry Rev 2006; 2: 235-44.

26. Ministerio de Salud de Chile. Guía clínica AUGE Depresión en personas de 15 y más. Series guías clínicas Minsal. Santiago de Chile: Minsal; 2013.

27. Alvarado R, Rojas G. El programa nacional para el diagnóstico y tratamiento de depresión en atención primaria: una evaluación necesaria. Rev Med Chile 2011; 139: 592-9.

28. Thase ME: The multifactorial presentation of depression acute care. J Clin Psychiatry 2013; 74 (suppl 2): 3-8.

29. Roca M, Monzón S, Vives M, López-Navarro E, Garcia-Toro M, Vicens C, et al. Cognitive function after clinical remission in patients with melancholic and non-melancholic depression: a 6 month follow-up study. J Affect Disord 2015; 171: 85-92.

30. Baumeister H, Parker G. Meta-review of depressive subtiping models. J Affect Disord 2012; 139: 126-40.

31. Parker G, Fink M, Shorter E, Taylor MA, Akiskal H, Berrios G, et al. Issues for DSM-5 whither melancholia? The case for its classification as a distinct mood disorder. Am J Psychiatry 2010; 167: 745-7.

32. American Psychiatric Association: Practice Guideline for the treatment of patients with major depressive disorder. Third Edition. Washington, DC: American Psychiatric Association; 2010.

33. Rakofsky JJ, Schettler PJ, Kinkead BL, Frank E, Judd LL, Kupfer DJ, et al. The prevalence and severity of depressive symptoms along the spectrum of unipolar depressive disorders: a post hoc analysis. J Clin Psychiatry 2013; 74 (11): 1084-91.

34. Eaton WW, Shao H, Nestadt G, Lee HB, Bienvenu OJ, Zandi P. Population-based study of first onset and chronicity in major depressive disorder. Arch Gen Psychiatry 2008; 65 (5): 513-20.

35. Skodol A, Grilo C, Reyes K, Geier T, Grant B, Hasin D. Relationship of personality disorders to the course of major depressive disorder in a nationally representative sample. Am J Psychiatry 2011; 168: 257-64.

36. Wilkinson P, Dubicka B, Kelvin R, Roberts Ch, Goodyer I. Treated depression in adolescents: predictors of outcome at 28 weeks. BJP 2009, 194: 334-41.

37. Kessler RC, Bromet EJ. The epidemiology of depression across cultures. Annu Rev Public Health 2013; 34: 11938.

38. Williams MD, Harris R, Dayan CM, Evans J, Gallache J, Ben-Shlomo Y. Thyroid function and the natural history 
of depression: findings from the Caerphilly Prospective Study (CaPS) and a meta-analysis. Clin Endocrinol 2009; 70: 484-92.

39. Bunevicius R, Prange A. Thyroid disease and mental disorders: cause and effect or only comorbidity? Curr Opin Psychiatry 2010; 23 (4): 363-8.

40. Moussavi S, Chatterji S, Verdes E, Tandon A, Patel V. Ustun B. Depression, chronic diseases, and decrements in health: results from the World Health Surveys. Lancet 2007; 370: 851-8.

41. Ministerio de Salud de Chile. Metas 2011-2020. Estrategia nacional de salud para el cumplimiento de los objetivos sanitarios de la década 2011-2020. Santiago de Chile: Minsal; 2011.

42. American Pychiatric Association. Manual Diagnóstico y Estadístico de los Trastornos Mentales DSM-5. 5 edición. Madrid: Editorial Médica Panamericana; 2014.

43. Koukopoulos A, Sani G. DSM-5 criteria for depression with mixed features: a farewell to mixed depression. Acta Psychiatr Scand 2014; 129 (1): 4-16.

44. National Institute for Health and Clinical Excellence. National Collaborating Centre for Mental Health. Depression the treatment and management of depression in adults. National Clinical Practice Guideline 90. London: NICE; 2010.

45. Ciudad A, Álvarez E, Roca M, Baca E, Caballero L, García de Polavieja P, et al. Early response and remission as predictors of a good outcome of a major depressive episode at 12-month follow-up. J Clin Psychiatry 2012; 73 (2): 185-91.

46. Rush A, Wisniewski S, Zisook S, Fava M, Sung S, Haley $\mathrm{C}$, et al. Is prior course of illness relevant to acute or longer-term outcomes in depressed out-patients? A STAR ${ }^{\star}$ D report. Psychol Med 2012; 42: 1131-49.

47. Stegenga BT, Geerlings MI, Torres-González F, Xavier M, Svab I, Penninx BW, et al. Risk factors for onset of multiple or long major depressive episodes versus single and short episodes. Soc Psychiatr Epidemiol 2013; 48 (7): 1067-75.

48. De Jonge P, Conradi HJ, Kaptein KI, Bockting CL, Korf J, Ormel J. Duration of subsequent episodes and periods of recovery in recurrent major depression. J Affect Disord 2010; 125 (1-3): 141-5.

49. Salvo L. Magnitud, impacto y estrategias de enfrentamiento de la depresión, con referencia a Chile. Rev Med Chile 2014; 142: 1157-64. 